\title{
HIPERTENSÃO ARTERIAL EM PACIENTES EM TRATAMENTO HEMODIALÍTICO E FATORES ASSOCIADOS
}

Júlia Sosa Antunes Cândido', Clarice Santana Milagres², Andréia Guerra Siman³, Camilo Amaro de Carvalho ${ }^{4}$, Marilane de Oliveira Fani Amaro ${ }^{5}$

${ }^{1}$ Enfermeira. Instituto de Nefrologia do Hospital Nossa Senhora das Dores. Ponte Nova-MG-Brasil.

${ }^{2}$ Enfermeira. Doutoranda em Saúde Coletiva. Universidade Estadual de Campinas. Campinas-SP-Brasil.

${ }^{3}$ Enfermeira. Mestre em Enfermagem. Professora da Universidade Federal de Viçosa. Viçosa-MG-Brasil.

${ }^{4}$ Farmacêutico. Doutor em Biologia Celular e Estrutural. Professor da Universidade Federal de Viçosa. Viçosa-MG-Brasil.

${ }^{5}$ Enfermeira. Doutora em Biologia Celular e Estrutural. Professora da Universidade Federal de Viçosa. Viçosa-MG-Brasil.

RESUMO: Estudo transversal, de caráter descritivo-exploratório. Objetivou analisar a presença de hipertensão arterial em pacientes portadores de doença renal crônica em tratamento hemodialítico e seus fatores associados. Foram entrevistados 140 pacientes de um instituto de nefrologia localizado no interior do estado de Minas Gerais, no período de agosto a outubro de 2013. A prevalência de hipertensão arterial nos pacientes portadores de doença renal crônica foi de $78,8 \%$. Constatou-se relação entre diabetes mellitus, dislipidemias e desconhecimento acerca da insuficiência renal com a hipertensão arterial. Identificou-se que $72,2 \%$ dos participantes excederam ao peso ideal, e destes, $63,3 \%$ apresentaram crise hipertensiva no período interdialítico. Conclui-se que a hipertensão arterial está presente significativamente nos indivíduos e os fatores associados são: adesão efetiva ao tratamento dialítico e medicamentoso; controle de ingesta hídrica e alimentar; e atividade física.

DESCRITORES: Enfermagem; Insuficiência renal crônica; Hipertensão.

\section{ARTERIAL HYPERTENSION IN PATIENTS RECEIVING HEMODIALYSIS TREATMENT AND ASSOCIATED FACTORS}

\begin{abstract}
This transversal study, with a descriptiveexploratory character, aimed to analyze the presence of arterial hypertension in patients with chronic kidney disease undergoing hemodialysis treatment, and its associated factors. Interviews were held with 140 patients of an institute of nephrology located in the rural area of the state of Minas Gerais, in August - October 2013. The prevalence of arterial hypertension in patients with chronic kidney disease was $78.8 \%$. A relationship was observed between diabetes mellitus, dyslipidemias and ignorance regarding renal failure with arterial hypertension. It was identified that $72.2 \%$ of the participants were above their ideal weight, and that of these, $63.3 \%$ presented hypertensive crisis in the interdialytic period. It is concluded that arterial hypertension is significantly present in individuals, and that the associated factors are: effective adherence to the dialysis and drug treatment; control of fluid and food intake; and physical activity.
\end{abstract}

DESCRIPTORS: Nursing; Chronic kidney disease; Hypertension.

\section{HIPERTENSIÓN ARTERIAL EN PACIENTES EN TRATAMIENTO HEMODIALÍTICO Y FACTORES ASOCIADOS}

RESUMEN: Estudio transversal, de carácter descriptivo exploratorio, cuyo objetivo fue analizar la presencia de hipertensión arterial en pacientes con enfermedad renal crónica en tratamiento hemodialítico y sus factores asociados. Fueron entrevistados 140 pacientes de un instituto de nefrología ubicado en el interior del estado de Minas Gerais, en el periodo de agosto a octubre de 2013. La prevalencia de hipertensión arterial en los pacientes con enfermedad renal crónica fue de $78,8 \%$. Se constató relación entre diabetes mellitus, dislipidemias y desconocimiento acerca de la insuficiencia renal con la hipertensión arterial. Se identificó que 72,2\% de los participantes excedieron al peso ideal y, de estes, $63,3 \%$ presentaron crisis hipertensiva en el periodo interdialítico. Se concluye que la hipertensión arterial está presente de modo significativo en los individuos y los factores asociados son: adhesión efectiva al tratamiento dialítico y medicamentoso; control de ingesta hídrica y alimentar; y actividad física.

DESCRIPTORES: Enfermería; Insuficiencia renal crónica; Hipertensión.
Autor Correspondente:

Marilane de Oliveira Fani Amaro

Universidade Federal de Viçosa

Av. Peter Henry Rolfs, s/no - 36570-000 - Viçosa-MG-Brasil

E-mail: marilaneamaro@yahoo.com.br
Recebido: 13/02/2015 Finalizado: 12/05/2015 


\section{INTRODUÇÃO}

Segundo o censo de diálise de 2012, estima-se que haja mais de 97 mil pacientes em tratamento dialítico no Brasil. Em 2011, este número foi de 91.314 pacientes, o que demonstra um número crescente de atendimento a esta especialidade ${ }^{(1-2)}$. Cerca de $60 \%$ dos pacientes em diálise no Brasil, apresentam hipertensão arterial sistêmica (HAS) ou diabetes como causas da evolução contínua e irreversível da perda da função renal por nefroesclerose hipertensiva e nefropatia diabética, respectivamente ${ }^{(3)}$.

A morbimortalidade em pacientes dialíticos tem como principais causas as doenças cardiovasculares. A mortalidade cardiovascular de pacientes em hemodiálise é geralmente alta, chegando a 50\% dos pacientes renais crônicos, sendo 10 a 20 vezes superior ao restante da população ${ }^{(4)}$. A suposta influência da HAS nestes índices tem gerado maior interesse nas pesquisas, dentro dos diferentes aspectos deste grupo característico, uma vez que seu surgimento está intimamente relacionado a fatores de risco não modificáveis, exemplificados pela idade, sexo, raça/cor e história familiar, e a fatores de risco ambientais modificáveis, como sedentarismo e ausência de estilo de vida saudável, sobrepeso/obesidade, alimentação inadequada (excesso de sódio) ${ }^{(5-8)}$.

Vale mencionar, que portadores da doença renal crônica (DRC) em tratamento de substituição renal necessitam adequar-se a uma dieta diferenciada, assim como aderir ao tratamento medicamentoso. A farmacoterapia envolvida no tratamento com intenção de estabilizar a doença, a fim de prevenir complicações e interferências no prognóstico desses indivíduos é constituída por medicamentos de uso contínuo e horários diversos ${ }^{(9)}$.

Na literatura, encontram-se estudos que avaliam as limitações (físicas, restrições laborais e perdas sociais) envolvidas no cotidiano dos pacientes renais crônicos $^{(10)}$ e que buscam compreender fatores relacionados à qualidade de vida (estágio da doença, componente físico e mental, hospitalização e tempo de diálise) e às comorbidades (hipertensão arterial e estresse) que influenciam a saúde e o cotidiano desses pacientes ${ }^{(11-12)}$.

Portanto, a prevalência da hipertensão arterial associada a outros fatores ainda tem fomentado pesquisas. A partir das considerações apresentadas, este estudo objetivou analisar a presença da hipertensão arterial em pacientes portadores de DRC em tratamento hemodialítico e seus fatores associados.

\section{MÉTODO}

Pesquisa transversal, de caráter descritivoexploratório, conduzido em um instituto de nefrologia de um hospital no interior deMinas Gerais, Brasil, no período de agosto a outubro de 2013.

A população alvo deste estudo foram os indivíduos portadores de DRC em terapia de substituição renal (hemodiálise) cadastrados no instituto de nefrologia, cenário de pesquisa deste estudo, nos três turnos de tratamento. De acordo com a rotina do serviço, os portadores da doença se dividiam no turno matutino e vespertino, de segunda a sábado; e o noturno, as segundas, quartas e sextas-feiras.

Estavam cadastrados 142 pacientes no instituto no período da coleta de dados. Para o fechamento amostral foi adotado critérios de inclusão: indivíduos portadores de DRC em hemodiálise, cadastrados no instituto, com idade igual ou superior a 18 anos, finalizando um total de 140 participantes. Ressalta-se a exceção de duas pessoas, pois, um negou-se a participar e outro se encontrava hospitalizado.

A coleta de dados foi realizada por meio de entrevistas com um roteiro semiestruturado, com a maioria das perguntas fechadas e pré-codificadas. O questionário foi aplicado diretamente ao paciente no próprio instituto de nefrologia.

A variável dependente analisada foi a presença de HAS, definida pela Sociedade Brasileira de Hipertensão pela VI Diretrizes Brasileiras de Hipertensão Arterial como uma condição clínica multifatorial, caracterizada por níveis elevados e sustentados de pressão arterial, apresentando pressão sistólica maior ou igual 140 mmHg e a pressão diastólica maior ou igual a $90 \mathrm{mmHg}^{(13)}$.

As variáveis independentes analisadas foram: características sociais: sexo, idade escolaridade e etnia; indicadores de condição de saúde: história de diabetes, dislipidemias e presença de picos hiper/hipotensivos; indicadores comportamentais/ hábitos de vida: consumo tabaco e álcool e realização de atividade física; indicadores do tratamento hemodialítico: conhecimento da medicação que faz uso e do horário da medicação, realização do controle da quantidade de líquido ingerido diariamente, tempo em 
que realiza hemodiálise (anos), frequência de sessões por semana (2, 3 ou 4 vezes), tipo de acesso vascular (fístula arteriovenosa-FAV, cateter de duplo-lúmen-CDL e Permcath), tempo de tratamento em anos (menor que 1, de 1 a 5, 6 a 10 e 10 anos e mais), crise hipertensiva durante o tratamento na máquina e durante o período interdialítico, peso seco (habitual) e ganho de peso no período interdialítico.

Para o armazenamento dos dados foi utilizado o software Office, Excel versão 2007 e para análise de dados foi utilizado o software Stata versão 9.0. A normalidade da distribuição das variáveis quantitativas foi avaliada usando o teste de Kolmogorov-Smirnov. Análise descritiva das variáveis foi apresentada por meio de medidas de tendência central e de variabilidade adequadas, bem como distribuição de frequências.

A associação entre as variáveis independentes e a presença de HAS na análise bivariada foi avaliada pelo teste qui-quadrado de Pearson $\left(x^{2}\right)$. O nível de significância adotado em todas as comparações foi de $a=5 \%$.

O estudo foi aprovado pelo Comitê de Ética em Pesquisa com Seres Humanos da Universidade Federal de Viçosa, parecer $\mathrm{n}^{\circ} 367.004$ e os preceitos éticos obedeceram à resolução 466/12 do Conselho Nacional de Saúde que subsidiou a elaboração do Termo de Consentimento Livre e Esclarecido (TCLE) que foi assinado previamente por todos os participantes do estudo. Foi solicitado, previamente, à Diretoria do Instituto de Nefrologia autorização para a realização do estudo, recebendo parecer favorável da Comissão de Ética em Pesquisa deste local.

\section{RESULTADOS}

Dentre os 140 pacientes do estudo, 55,3\% eram do sexo masculino. A média da idade foi de 55,79 anos ( $\mathrm{dp}= \pm 16,72$ anos) com a faixa etária de 40 a 59 anos e 60 a 79 anos correspondendo a $36,15 \%$ e $33,08 \%$, respectivamente.

A prevalência global de HAS nos pacientes portadores de DRC foi de $78,8 \%$, correspondendo a 104 pacientes da amostra estudada. Em relação às variáveis sociais, não foi possível observar diferença significantenaprevalência dehistória deHAS(Tabela 1).

A Tabela 2 apresenta variáveis de hábitos de vida e condições de saúde. Pode-se observar comorbidades nos pacientes pesquisados, com
Tabela 1 - Prevalência de HAS, segundo variáveis sociodemográficas de pacientes. Minas Gerais-Brasil, 2013

\begin{tabular}{|c|c|c|c|}
\hline Variáveis & $\mathbf{N}$ & $\begin{array}{c}\text { Prevalência } \\
(\%)\end{array}$ & $\mathbf{p}^{* *}$ \\
\hline \multicolumn{4}{|l|}{ Sexo } \\
\hline Feminino & 48 & 81,4 & \multirow{2}{*}{0,52} \\
\hline Masculino & 56 & 76,7 & \\
\hline \multicolumn{4}{|l|}{ Faixa etária } \\
\hline $20-39$ & 25 & 83,3 & \multirow{4}{*}{0,63} \\
\hline $40-59$ & 37 & 76,6 & \\
\hline $60-79$ & 33 & 74,4 & \\
\hline 80 anos e mais & 09 & 90 & \\
\hline \multicolumn{4}{|l|}{ Etnia } \\
\hline Caucasiano & 33 & 75 & \multirow{3}{*}{0,73} \\
\hline Pardo & 35 & 79,5 & \\
\hline Negro & 36 & 81,8 & \\
\hline \multicolumn{4}{|l|}{$\begin{array}{l}\text { Escolaridade } \\
\text { (anos) }\end{array}$} \\
\hline Até 4 & 67 & 78,2 & 0,74 \\
\hline 5 a 9 & 27 & 81,8 & \\
\hline 10 a 12 & 07 & 77,8 & \\
\hline Mais de 12 & 03 & 60 & \\
\hline
\end{tabular}

* Número de indivíduos na amostra / ** Teste $\mathrm{x}^{2}$ de Pearson

maior prevalência de diabetes $(91,7 \%)$ e uma totalidade de indivíduos que apresentam história de HAS acompanhado do quadro de dislipidemia, assim como aqueles que desconheciam essa condição de doença. Logo, foi possível observar associações estatisticamente significantes entre a presença dessas doenças e a ocorrência de história de HAS nos pacientes portadores de DRC. Houve maiores prevalências na ausência de comorbidades, hábitos de ingerir bebidas alcoólicas e hábito de fumar $(79,6 \%$, $79,7 \%$ e $80 \%$ respectivamente) (Tabela 2 ).

Entre os indivíduos investigados, aqueles que autorreferiram não conhecer a medicação que utilizavam, correspondeu a $87,8 \%$. No entanto, $79,5 \%$ disseram conhecer o horário de uso dos medicamentos utilizados. Ainda em relação à medicação, dentre os indivíduos que apresentam picos hipertensivos, 61,5\% relatam ter saído do esquema medicamentoso prescrito, apresentando as justificativas: dificuldade de adesão ao processo terapêutico, grande quantidade de medicamentos, falta de compreensão quanto à importância da terapia e não aceitação dos eventos adversos.

Quanto aos episódios de crise hipertensiva, este resultado foi estatisticamente significante, conforme observado na Tabela 3. 
Tabela 2 - Prevalência de HAS, segundo variáveis de hábitos de vida e condições de saúde, de pacientes. Minas Gerais-Brasil, 2013

\begin{tabular}{|c|c|c|c|}
\hline Variáveis & $\mathbf{N}$ & $\begin{array}{c}\text { Prevalência } \\
(\%)\end{array}$ & $\mathbf{p}^{* *}$ \\
\hline \multicolumn{4}{|l|}{ História de diabetes } \\
\hline Não & 71 & 74 & \multirow{2}{*}{0,02} \\
\hline Sim & 33 & 91,7 & \\
\hline \multicolumn{4}{|l|}{ Faixa etária } \\
\hline Não & 78 & 73,6 & \multirow{3}{*}{0,01} \\
\hline Sim & 15 & 100 & \\
\hline Desconhece & 11 & 100 & \\
\hline \multicolumn{4}{|c|}{ Presença de outras comorbidades } \\
\hline Nenhuma & 74 & 79,6 & \multirow{4}{*}{0,41} \\
\hline $\begin{array}{l}\text { Perda de } \\
\text { transplante renal } \\
\text { prévio }\end{array}$ & 02 & 50 & \\
\hline $\begin{array}{l}\text { Doenças } \\
\text { cardiovasculares }\end{array}$ & 07 & 70 & \\
\hline Outras & 21 & 84 & \\
\hline \multicolumn{4}{|l|}{ Hábito de fumar } \\
\hline Não & 88 & 78,6 & \multirow{2}{*}{0,89} \\
\hline $\operatorname{Sim}$ & 16 & 80 & \\
\hline \multicolumn{4}{|c|}{ Hábito de beber bebidas alcoólicas } \\
\hline Não & 98 & 79,7 & \multirow{2}{*}{0,36} \\
\hline Sim & 06 & 66,7 & \\
\hline \multicolumn{4}{|c|}{ Pratica exercício físico } \\
\hline Sim & 27 & 69,2 & \multirow{2}{*}{0,08} \\
\hline Não & 77 & 82,8 & \\
\hline
\end{tabular}

* Número de indivíduos na amostra / ** Teste $x^{2}$ de Pearson

Na pesquisa realizada, foi calculado, para cada participante, o ganho de peso ideal em relação ao seu peso seco. O pico hipertensivo observado durante a sessão de hemodiálise é causado pela sobrecarga de líquidos e sódio, ocorrendo retenção (edema) e surgimento da HAS. Após a realização da ultrafiltração na máquina haverá redução de líquidos retidos, levando à normalização da pressão arterial.

A Figura 1 apresenta a distribuição de ganho de peso dos indivíduos no período interdialítico, na qual $72,2 \%$ excederam ao peso ideal e destes, 63,3\% apresentaram picos hipertensivos. O cálculo de ganho de peso dos indivíduos no período interdialítico foi observado associandose o ganho de peso e aparecimento de picos hipertensivos. Dentre os $16,4 \%$ de indivíduos que não excederam o peso, 43,4\% apresentaram picos hipertensivos, enquanto aqueles indivíduos
Tabela 3 - Prevalência de HAS, segundo variáveis relacionadas ao tratamento hemodialítico de pacientes. Minas Gerais-Brasil, 2013

\begin{tabular}{|c|c|c|c|}
\hline Variáveis & $\mathbf{N}$ & $\begin{array}{c}\text { Prevalência } \\
(\%)\end{array}$ & $\mathbf{p}^{* *}$ \\
\hline \multicolumn{4}{|c|}{ Conhecimento da medicação que faz uso } \\
\hline Sim & 68 & 75,6 & \multirow{2}{*}{0,11} \\
\hline Não & 36 & 87,8 & \\
\hline \multicolumn{4}{|c|}{ Conhecimento do horário da medicação que faz uso } \\
\hline Sim & 101 & 79,5 & \multirow{2}{*}{0,83} \\
\hline Não & 03 & 73 & \\
\hline \multicolumn{4}{|c|}{ Controle da quantidade de líquido ingerida diariamente } \\
\hline Sim & 75 & 75,8 & \multirow{2}{*}{0,14} \\
\hline Não & 29 & 87,9 & \\
\hline
\end{tabular}

Tempo em que realiza hemodiálise (anos)

\begin{tabular}{|c|c|c|}
\hline Até 1 & 32 & 82,1 \\
\hline 1 a 5 & 40 & 80 \\
\hline 6 a 10 & 21 & 75 \\
\hline Mais de 10 & 11 & 73,3 \\
\hline
\end{tabular}

Número de sessões por semana

\begin{tabular}{|c|c|c|c|}
\hline Duas & 04 & 66,7 & \multirow{3}{*}{0,45} \\
\hline Três & 96 & 79 & \\
\hline Quatro & 04 & 100 & \\
\hline \multicolumn{4}{|c|}{ Tipo de acesso vascular } \\
\hline FAV & 97 & 77,6 & \multirow{3}{*}{0,37} \\
\hline Permcath & 03 & 100 & \\
\hline $\mathrm{CDL}$ & 04 & 100 & \\
\hline
\end{tabular}

Crise hipertensiva durante o tratamento na máquina

\begin{tabular}{|c|c|c|c|}
\hline Não & 37 & 67,3 & 01 \\
\hline Sim & 67 & 87 & $<0,01$ \\
\hline
\end{tabular}

Crise hipertensiva durante o período interdialítico

\begin{tabular}{lll}
\hline Não & 43 & 68,5 \\
\hline Sim & 53 & 97,9 \\
\cline { 1 - 2 } Desconhece & 08 & 72,7
\end{tabular}$<0,01$

* Número de indivíduos na amostra / ** Teste $x^{2}$ de Pearson

que não recordaram o peso médio de ganho (11,4\%), 62,5\% apresentaram picos hipertensivos.

Em relação aos hábitos de vida dos indivíduos em tratamento hemodialítico, 53 participantes relataram apresentar picos hipertensivos no período interdialítico. A Figura 2 apresenta a associação desse pico hipertensivo nos 53 participantes e hábito de tabagismo; desuso da medicação necessária ao tratamento; ausência de controle da ingestão hídrica; uso de bebidas alcoólicas e prática de atividade física. 


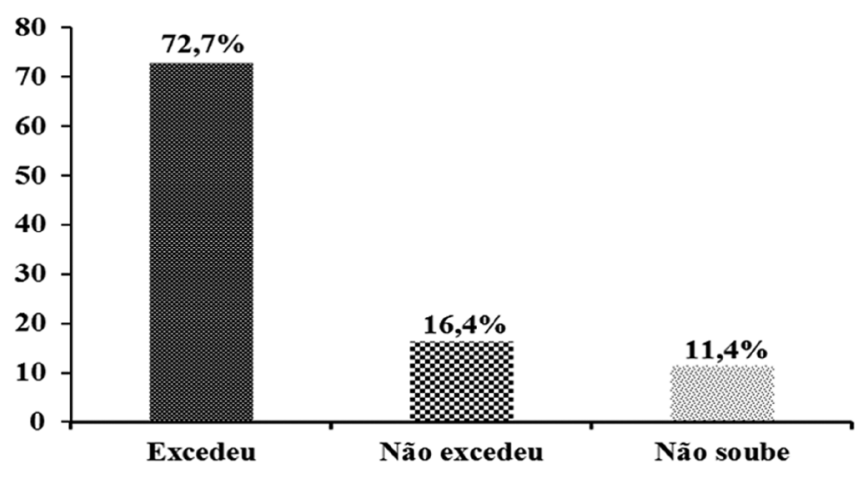

Figure 1 - Distribution of weight gain of the patients with CKD in the interdialytic period. Minas Gerais-Brazil, 2013

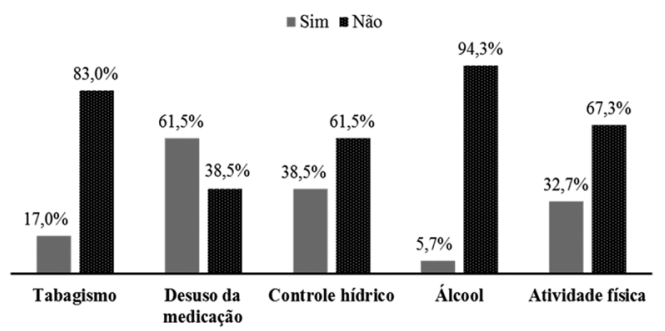

Figure 2 - Living habits of the patients with CKD who presented hypertensive peaks in the interdialytic period. Minas Gerais-Brazil, 2013

\section{DISCUSSÃO}

Pacientes em tratamento hemodialítico apresentam, como complicações comuns, os eventos cardiovasculares, sendo a HAS a principal. Como consequência há uma aceleração no processo de formação da aterosclerose e aumento dos riscos de acidentes cardiovasculares, aneurismas e insuficiência cardíaca ${ }^{(4,13-15)}$.

O controle dos níveis pressóricos é possivelmente a medida de maior impacto para a prevenção da progressão do dano renal, além do controle adequado de outras situações clínicas como a diabetes, a insuficiência cardíaca, a anemia, ou seja, as principais comorbidades que acometem os pacientes renais crônicos ${ }^{(13-15)}$.

Estudos tem mostrado também, que o ganho de peso interdialítico traz complicações. Para manter um peso ideal, o ganho de peso interdialítico não deve ultrapassar cerca de $3 \%$ do peso seco de cada indivíduo ${ }^{(8)}$. Os resultados encontrados mostraram que $72,2 \%$ dos participantes excederam em média o peso interdialítico adequado em relação ao seu peso seco. Destaca-se que entre os indivíduos que apresentaram picos hipertensivos, 61,5\% não controlavam a ingesta hídrica, logo, estavam acima do peso ideal, o que contribui para descontrole da pressão arterial e outras complicações como insuficiência congestiva e até morte ${ }^{(7-8)}$.

O peso seco é estimado pelo médico a partir da avaliação dos sinais clínicos de hidratação e comportamento dos níveis pressóricos, e é um dado indispensável para a determinação do volume a ser retirado na sessão de hemodiálise ${ }^{(8)}$. Por meio do peso seco, da diurese residual e da quantidade da ingesta hídrica, a enfermagem é capaz de controlar o balanço hídrico e avaliar a hidratação dos indivíduos ${ }^{(7,16)}$.

Alguns estudos apontam que as principais causas dos pacientes não cumprirem os aconselhamentos sobre o controle de peso é a pouca informação e a falta de compreensão acerca das verdadeiras necessidades de restringir sódio e água, ou por uma falta de clareza do que é considerado líquido na dieta ${ }^{(17-18)}$.

A enfermagem, diante da orientação sobre o autocuidado e prescrição de enfermagem, tem o papel de conscientizar o indivíduo quanto à manutenção do peso na preservação de sua saúde e bem-estar, ressaltando os riscos de excesso hídrico e de morte prematura por complicações cardiovasculares ${ }^{(10,12,15,19-22)}$. Nesse aconselhamento, deve-se esclarecer que alguns alimentos como café, chá, sopa, sorvete, água de 
coco, melancia, abacaxi, laranja, tomate, alface, dentre outros, são ricos em água e devem ser incluídos no volume total de líquidos ingeridos, pois a maioria, não é considerada como parte da ingesta hídrica, acarretando a aumento da volemia e consequentemente à $\operatorname{HAS}^{(15,23)}$.

Quanto ao tratamento medicamentoso do indivíduo com DRC, este se baseia no uso de hipotensores ${ }^{(9,24)}$. Portadores de DRC devem ter rigoroso controle medicamentoso e dietético, controlados diariamente, cuja finalidade é estabilizar o estado de saúde do doente, especialmente, quando se trata de $\mathrm{HAS}^{(9)}$. Entretanto, uma adesão efetiva implica ao doente se adequar com habilidade em cumprir as recomendações clínicas, ingerir adequadamente o medicamento prescrito, seguir o estilo de vida saudável e realizar os procedimentos de monitoramento frequentemente ${ }^{(9,22)}$. A aderência ao esquema medicamentoso deve ir além da prescrição, envolvendo o indivíduo no autocuidado por meio de aconselhamentos e educação em saúde, sendo essas ações realizadas pelo profissional de enfermagem, uma vez que está em constante acompanhamento ao indivíduo ${ }^{(15,25)}$.

Em relação aos demais hábitos de vida, um fator encontrado nesta pesquisa foi que, dentre os participantes que apresentaram picos hipertensivos, somente $17 \%$ eram tabagistas e $5 \%$ faziam uso de bebida alcoólica, não apresentando correlação entre tabagismo e uso de bebida alcoólica com aparecimento de picos hipertensivos.

Segundo a VI Diretriz Brasileira de Hipertensão Arterial, o consumo baixo a moderado de álcool e o tabagismo não estão absolutamente definidos como fatores de risco para a HAS. Há evidências de que, a ingestão de álcool por períodos prolongados de tempo pode aumentar a pressão arterial. E, a cessação do tabagismo constitui medida fundamental e prioritária na prevenção, contudo, não há evidências de que, para o controle de PA, haja benefícios ${ }^{(13)}$.

Entretanto, o tabagismo e o excesso do uso de bebida alcoólica devem ser intensamente combatidos, pois são fatores de risco às doenças cardiovasculares modificáveis, dependentes expressamente do comportamento do indivíduo ${ }^{(12-13,24-25)}$.
Dentre os participantes que apresentaram picos hipertensivos, 67,3\% relataram não praticar nenhuma atividade física. A prática regular de exercícios físicos é recomendada para todos os portadores de HAS, inclusive aqueles sob tratamento medicamentoso, pois diminui a pressão arterial sistólica/diastólica em 6,9/4,9 $\mathrm{mmHg}$. A atividade física também traz benefícios aos aspectos fisiológicos e metabólicos como o aumento na massa óssea, no aumento do colesterol High Density Lipoproteins (HDL), na flexibilidade e na força, ajudando a controlar e a prevenir o diabetes, as dislipidemias, a obesidade, auxiliando na diminuição do risco de desenvolver doenças cardiovasculares ${ }^{(26-27)}$.

Há os prejuízos musculoesqueléticos, que estão entre os principais fatores que alteram a qualidade de vida do indivíduo com DRC, principalmente os de tratamento de longa duração ${ }^{(15)}$. Esclarecendo, o indivíduo com DRC apresenta distúrbios do metabolismo mineral. Dentre eles, está a elevação do nível de paratormônio (PTH), que aumentado leva à mobilização óssea de cálcio culminando em doenças ósseas descritas pelos nefrologistas como osteodistrofia renal, na qual gera limitações físicas levando a dificuldade em caminhar, fazer esforço físico, subir escadas, correr, entre outras $^{(27-29)}$.

Neste contexto, a literatura reporta ser necessário que os profissionais de saúde aconselhem os pacientes quanto a outros métodos de prevenção referente à HAS, refletindo diretamente no pico hipertensivo interdialítico, como à adesão dos medicamentos, ingesta hídrica moderada, redução da ingestão de sódio, sendo fundamental a participação da enfermagem nestas intervenções ${ }^{(8,12,19-24)}$.

De forma consistente, destaca-se a importância de inserir a educação em saúde no ambiente hospitalar, valorizando a sua relevância em toda a organização de saúde, a fim de que os indivíduos possam optar por decisões saudáveis, capazes de contribuir com a sua qualidade de $v i^{2} a^{(7,29)}$. Em decorrência disto, indivíduos com conhecimentos efetivos são mais equilibrados e aderem ao tratamento de forma mais eficaz, reduzindo complicações como os eventos cardiovasculares $^{(4,13-15)}$.

Torna-se, portanto, essencial que a enfermagem realize educação em saúde com os pacientes 
em tratamento hemodialítico para que esses tenham conhecimento a respeito de seu quadro clínico e de seu tratamento de forma dinâmica, e assim, adquiram segurança e subsídios para seu autocuidado ${ }^{(29-30)}$. Além disso, a enfermagem atuante nos serviços de diálise deve envolverse ao máximo, participando não apenas do tratamento técnico, mas também, em relação ao indivíduo junto à sua família, ao seu ambiente, promovendo qualidade de vida.

\section{CONSIDERAÇÕES FINAIS}

A DRC altera de forma significativa as condições de vida do indivíduo, com necessidade de adesão efetiva ao tratamento dialítico, medicamentoso, ingesta hídrica, alimentos controlados e atividade física. Entretanto, principalmente, a ingesta hídrica inadequada, não adesão ao tratamento e sedentarismo agravam o estado clínico.

Contudo, as condições apontadas se caracterizam como modificáveis, cabendo ao profissional de enfermagem interagir e acompanhar os indivíduos na mudança de hábitos, identificando as deficiências comportamentais, assim como orientá-lo de maneira efetiva, garantindo melhor condições de vida e sobrevida ao paciente.

São indispensáveis as terapêuticas educativas para que o indivíduo se torne mais seguro quanto ao seu autocuidado e corresponsável pela sua saúde, consequentemente, ativo em seu tratamento. Cabe ao profissional de enfermagem auxiliá-lo na sua terapêutica diária, mediante avaliação e aconselhamento.

Conhecer a prevalência de HA e seus fatores associados em pacientes portadores de DRC é imprescindível, pois pode estimar a extensão do problema neste grupo, e desta forma, favorecer a condução de ações eficazes e que resultem em maior expectativa de vida na população assistida. Com relação às limitações do estudo, destaca-se que, embora contemple pacientes renais em tratamento hemodialítico, foi realizado em apenas um município, o que implica que as generalizações devem ser realizadas com cautela. Novos estudos devem ser realizados para uma maior generalização dos resultados aqui encontrados.

\section{REFERÊNCIAS}

1. Sociedade Brasileira de Nefrologia. Censo de diálise SBN 2012 [Internet]. [acesso em 09 nov 2013]. Disponível: http://www.sbn.org.br/pdf/publico2012.pdf

2. Junior CSM, Mendonça RRS, Hatem RORF, Souza ALS, Chaves AR, Bastos MG, et al. O custo de soluções alcalinas em sessões de hemodiálise ambulatorial: uma análise sobre o desperdício a partir do controle dos processos. J. Bras. Nefrol. [Internet] 2014;36(4) [acesso em 04 jan 2014]. Disponível: http://dx.doi. org/10.5935/0101-2800.20140072

3. Sesso RC, Lopes AA, Thome FS, Lugon JR, Watanabe Y, Santos DR. Relatório do Censo Brasileiro de Diálise Crônica 2012. J. Bras. Nefrol. [Internet]. 2014;36(1) [acesso em 05 mai 2014]. Disponível: http://dx.doi. org/10.5935/0101-2800.20140009

4. Almeida FAA, Machado FC, Moura Junior JA, Guimarães AC. Global and cardiovascular mortality and risk factors in patients under hemodialysis treatment. Arq. Bras. Cardiol. [Internet]. 2010;94(2) [acesso em 04 jan 2014]. Disponível: http://dx.doi. org/10.1590/S0066-782X2010005000003

5. Santos ZMSA, Lima HP. Tecnologia educativa em saúde na prevenção da hipertensão arterial em trabalhadores: análise das mudanças no estilo de vida. Texto contexto enferm. [Internet]. 2008;17(1) [acesso em 04 jan 2014]. Disponível: http://dx.doi.org/10.1590/ S0104-07072008000100010

6. Custodio IL, Lima FET, Almeida MI, Silva LF, Monteiro ARM. Perfil sociodemográfico e clínico de uma equipe de enfermagem portadora de Hipertensão Arterial. Rev. bras. enferm. [internet]. 2011;64(1) [acesso em 04 jan 2014]. Disponível: http://dx.doi.org/10.1590/ S0034-71672011000100003

7. Carvalho GMC, Lima FET, Barbosa IV, Melo EM. Estudos brasileiros sobre nefrologia nas teses e dissertações de enfermagem. Rev. bras. enferm. [Internet]. 2010;63(6) [acesso em 05 jan 2014]. Disponível: http://dx.doi. org/10.1590/S0034-71672010000600028

8. Nerbass FB, Morais JG, Santos RG, Krüger TS, Koene TT, Luz Filho HA. Fatores relacionados ao ganho de peso interdialítico em pacientes em hemodiálise. J. Bras. Nefrol. [Internet]. 2011;33(3) [acesso em 06 mai 2014]. Disponível: http://dx.doi.org/10.1590/S010128002011000300005

9. Sgnaolin V, Figueiredo AEPL. Adesão ao tratamento farmacológico de pacientes em hemodiálise. J. Bras. Nefrol. [Internet]. 2012;34(2) [acesso em 04 jan 2014]. Diponível: http://dx.doi.org/10.1590/S010128002012000200002

10. Valle LS, Souza VF, Ribeiro AM. Estresse e ansiedade em 
pacientes renais crônicos submetidos à hemodiálise. Estud. Psicol. [Internet]. 2013;30(1) [acesso em 04 jan 2014]. Diponível: http://dx.doi.org/10.1590/S0103166X2013000100014

11. Pinto NA, Falcao EBM. Religiosidade no contexto médico: entre a receptividade e o silêncio. Rev. Bras. Educ. Med. [Internet]. 2014;38(01) [acesso em 04 jan 2015]. Disponível: http://dx.doi.org/10.1590/S010055022014000100006

12. Guerra-Guerrero V, Sanhueza-Alvarado O, CaceresEspina M. Qualidade de vida de pessoas em hemodiálise crônica: relação com variáveis sociodemográficas, médico-clínicas e de laboratório. Rev. Latino-Am. Enfermagem [Internet]. 2012;20(5) [acesso em 04 jan 2014]. Disponível: http://dx.doi.org/10.1590/S010411692012000500004

13. Sociedade Brasileira de Cardiologia/Sociedade Brasileira de Hipertensão/Sociedade Brasileira de Nefrologia. VI Diretrizes Brasileiras de Hipertensão. Arq Bras Cardiol [Internet] 2010;95(1supl.1) [acesso em 04 jan 2014]. Disponível: http://publicacoes.cardiol.br/ consenso/2010/Diretriz_hipertensao_associados.pdf

14. Pivatto DR, Abreu IS. Principais causas de hospitalização de pacientes em hemodiálise no município de Guarapuava, Paraná, Brasil. Rev. gauch. enferm. [Internet]. 2010;31(3) [acesso em 04 jan 2014]. Disponível: http://dx.doi.org/10.1590/S198314472010000300015

15. Santos I, Rocha RPF, Berardinelli LMM. Necessidades de orientação de enfermagem para o autocuidado de clientes em terapia de hemodiálise. Rev. bras. enferm. [Internet]. 2011;64(2) [acesso em 04 jan 2014]. Disponível: http://dx.doi.org/10.1590/S003471672011000200018

16. Burmeister JE, Mosmann CB, Costa VB, Saraiva RT, Grandi RR, Bastos JP,et al. Prevalência de fatores de risco cardiovascular em pacientes em hemodiálise - o estudo CORDIAL. Arq. Bras. Cardiol. [Internet]. 2014;102(5) [acesso em 04 jan 2014]. Disponível: http:// www.arquivosonline.com.br/2014/aop/aop_5855.pdf

17. Martins BTC, Ribeiro Junior E. Perguntas e respostas sobre a nutrição em diálise. São Paulo: RCN editora; 2008.

18. Oliveira SM, Ribeiro RCHM, Ribeiro DF, Lima LCEQ, Pinto MH, Poletti NAAP. Elaboração de um instrumento da assistência de enfermagem na unidade de hemodiálise. Acta paul. enferm. [Internet]. 2008;21(n. spel) [acesso em 04 jan 2014]. Disponível: http://dx.doi.org/10.1590/S0103-21002008000500006

19. Santos I, Rocha RPF, Berardinelli LMM. Qualidade de vida de clientes em hemodiálise e necessidades de orientação de enfermagem para o autocuidado. Esc. Anna Nery [Internet]. 2011;15(1) [acesso 04 jan 2014]. Disponível: http://dx.doi.org/10.1590/S1414-

\section{5}

20. Barbosa GS, Valadares GV. Hemodiálise: estilo de vida e a adaptação do paciente. Acta paul. enferm. [Internet]. 2009;22(n. spel) [acesso em 04 mai 2014]. Disponível: http://dx.doi.org/10.1590/S010321002009000800014

21. Madeiro AC, Machado PDLC, Bonfim IM, Braqueais AR, Lima FET. Adesão de portadores de insuficiência renal crônica ao tratamento de hemodiálise. Acta paul. enferm. [Internet]. 2010;23(4) [acesso em 05 mai 2014]. Disponível: http://www.scielo.br/pdf/ape/v23n4/16.pdf

22. Ribeiro RCH, Miranda ALL, Cesarino CB, Bertolin DC, Ribeiro DF, Kusumota L. Necessidades de aprendizagem de profissionais de enfermagem na assistência aos pacientes com fístula arteriovenosa. Acta paul. enferm. [Internet]. 2009;22(n.spel) [acesso em 04 mai 2014]. Disponível: http://dx.doi. org/10.1590/S0103-21002009000800012

23. Morais AAC, Silva Maria AT, Faintuch J, Vidigal EJ, Costa RA, Lyrio DC et al. Correlation of nutritional status and food intake in hemodialysis patients. Clinics [Internet]. 2005;60(3) [acesso em 05 mai 2014]. Disponível: http://dx.doi.org/10.1590/S180759322005000300002

24. Bastos MG, Bregman R, Kirsztajn GM. Doença renal crônica: frequente e grave, mas também prevenível e tratável. Rev. Assoc. Med. Bras. [Internet]. 2010;56(2) [acesso em 04 jan 2014]. Disponível: http://dx.doi. org/10.1590/S0104-42302010000200028

25. Mousinho PLM, Moura MES. Hipertensão Arterial: fatores relacionados à adesão do cliente com hipertensão ao tratamento medicamentoso. Saude Colet. [Internet] 2008;5(25) [acesso em 05 mai 2014]. Disponível: http://www.redalyc.org/ pdf/842/84225505.pdf

26. Nascimento LCA, Coutinho EB; Silva KNG. Efetividade do exercício físico na insuficiência renal crônica. Fisioter. mov. [Internet]. 2012;25(1) [acesso em 05 mai 2014]. Disponível: http://dx.doi.org/10.1590/S010351502012000100022

27. Soares KTA, Viesser MV, Rzniski TAB, Brum EP. Eficácia de um protocolo de exercícios físicos em pacientes com insuficiência renal crônica, durante o tratamento de hemodiálise, avaliada pelo SF-36. Fisioter. mov. [Internet]. 2011;24(1) [acesso em 05 mai 2014]. Disponível: http://dx.doi.org/10.1590/S010351502011000100015

28. Vieira WP, Gomes KWP, Frota NB, Andrade JECB, Vieira RMRA, Moura FEA et al. Manifestações musculoesqueléticas em pacientes submetidos à hemodiálise. Rev. Bras. Reumatol. [Internet]. 2005;45(6)[acesso em mai 2014]. Disponível: http:// dx.doi.org/10.1590/S0482-50042005000600005 
29. Figueira AB, Amestoy SC, Cecagno DTFS, Trindade $\mathrm{LL}$, Correa VA. Visão do enfermeiro frente à prática da educação em saúde no ambiente hospitalar. Cogitare enferm. [Internet] 2013;18(2) [acesso em 05 mai de 2014]. Disponível: http://dx.doi.org/10.5380/ ce.v18i2.32580

30. Queiroz MVO, Dantas MCQ, Ramos IC, Jorge MSB. Tecnologia do cuidado ao paciente renal crônico: enfoque educativo-terapêutico a partir das necessidades dos sujeitos. Texto contexto enferm. [Internet]. 2008;17(1)[acesso em 05 mai de 2914]. Disponível: http://dx.doi.org/10.1590/S010407072008000100006 